Classification

Physics Abstracts

$62.60-68.10$

\title{
Spectral functions for a semi-infinite liquid
}

\author{
V. R. Velasco and F. Garcia-Moliner \\ Instituto de Física del Estado Sólido (CSIC), Serrano 123, E-28006 Madrid, Spain
}

(Reçule 11 mars 1985, révisé le 15 mai, accepté le 17 juin 1985)

\begin{abstract}
Résumé. - Les contributions de surface à la densité d'états totale, que détermine la dépendance de la chaleur spécifique de surface dans la température, sont calculées d'une façon simple par l'application directe de la méthode de raccordement des fonctions de Green de surface au liquide non visqueux. Une différence sur le comportement de basse température est clarifiée et on montre la relation avec la théorie d'une autre fonction spectrale d'intérêt physique.

Abstract. - The surface contributions to the total density of states, which determine the temperature dependence of the surface specific heat, are very simply obtained by a direct application of the surface Green function matching analysis to an inviscid liquid. A discrepancy on the low temperature behaviour is resolved while showing the connection with the theory of another surface spectral function of physical interest.
\end{abstract}

There appears to be some discrepancies between separate theoretical results for the low temperature behaviour of the surface specific heat of liquid surfaces [1, 2]. Djafari-Rouhani and Dobrzynski [1] obtained an expression for the surface specific heat including several powers of $T$ whereas Iosilevskii [2] reported a $T^{2}$ behaviour at low temperatures. This depends on the frequency dependence of the different contributions to the density of states, which have been studied in detail in [1] by constructing the complete Green function of the semi-infinite fluid.

The density of states is an example of a spectral function in which the details corresponding to all the possible propagation directions have been included. An opposite example is the surface projected spectral density, which depends on frequency and on the wavevector projection onto the boundary surface. The latter spectral density constitutes the appropriate power function for the analysis of scattering of external probes by a stress free surface. A useful approach is based on working always with surface projections of the physical quantities relevant to the scattering process [3] and going directly to the surface projected spectral density. This has been recently reviewed in [4]. Another alternative is provided by the Surface Green Function Matching (SGFM) method [5], in which both spectral functions are obtained by working always with the surface projections of the Green functions, as explained in [5]. Fluid surfaces and interfaces have been studied in this way, including viscosity $[6,7]$.

For the specific heat problem one is interested in the inviscid fluid. This can be studied very simply in the SGFM analysis. Always within the frame of a phenomenological long wave theory, the equation of motion for a longitudinal wave of velocity $l$ is

$$
-l^{2} \nabla^{2} \phi+\partial^{2} \phi / \partial t^{2}=\psi_{\text {ext }},
$$


$\phi$ being the velocity potential and $\psi_{\text {ext }}$ an appropriate external potential generating the external driving force. The Green function associated to equation (1) is

$$
G(\mathbf{k}, \omega)=\left(l^{2} k^{2}-\omega^{2}\right)^{-1} .
$$

The boundary condition at the fluid surface $z=0$ is [8]

$$
\rho \partial^{2} \phi / \partial t^{2}=\gamma \frac{\partial}{\partial z}\left(\partial^{2} \phi / \partial x^{2}+\partial^{2} \phi / \partial y^{2}\right) \text {. }
$$

where $\gamma$ is the surface tension coefficient.

The Green function $\left(G_{\mathrm{s}}\right)$ of the surface problem has a surface projection $\mathcal{G}_{\mathrm{s}}$ given by the matching formula

$$
\mathcal{G}_{\mathrm{s}}^{-1}=-\mathcal{A}^{(-)} \mathcal{G}^{-1}+m_{\mathrm{s}}
$$

if the fluid occupies the half-space $z>0$. Here $A^{(-)}$is a differential operator including a linear combination of the surface projected Green function and its normal derivative [5] as obtained from the constitutive relations of the medium. $m_{\mathrm{s}}$ measures the effect of the extra force due to the surface tension. Using the formulae of $[5,7]$ for a given wavevector projection $\kappa$ onto the surface we have in this case

and

$$
\mathcal{G}=\left(2 l^{2} \beta_{l}\right)^{-1} ; \quad \beta_{l}=\left(\kappa^{2}-\omega^{2} / l^{2}\right)^{1 / 2}
$$

$$
m_{\mathrm{s}}=-l^{2} \rho \omega^{2} / \gamma^{2} \kappa^{2} ; \quad \mathcal{A}^{( \pm)} \doteq l^{2} \mathcal{G}^{( \pm)} ; \quad \mathcal{G}^{( \pm)}= \pm\left(2 l^{2}\right)^{-1} .
$$

For the total density of states per unit surface area it suffices to evaluate

where

$$
N_{\mathrm{t}}(\omega)=\frac{2 \omega}{(2 \pi)^{2}} \int \mathrm{d}^{2} \kappa N_{\mathrm{t}}\left(\kappa, \omega^{2}\right),
$$

$$
N_{\mathrm{t}}\left(\kappa, \omega^{2}\right)=\frac{1}{2} N_{\mathrm{B}}+H\left(\kappa, \omega^{2}\right)+S\left(\kappa, \omega^{2}\right),
$$

being

$$
\begin{gathered}
H=\frac{1}{2 \pi} \lim _{\eta \rightarrow 0^{+}} \frac{\partial \arg \mathcal{S}\left(\kappa, \omega^{2}-i \eta\right)}{\partial \omega^{2}}, \\
S=\frac{1}{\pi} \lim _{\eta \rightarrow 0^{+}} \frac{\partial \arg \mathcal{G}_{\mathrm{s}}^{-1}\left(\kappa, \omega^{2}-i \eta\right)}{\partial \omega^{2}}
\end{gathered}
$$

and $\frac{1}{2} N_{\mathrm{B}}$ denotes the bulk density of states.

It can be seen that

$$
H=-\frac{1}{4} \delta\left(\omega^{2}-\omega_{l}^{2}\right), \quad \omega_{l}=l \kappa,
$$

where $\omega_{l}$ is the bulk frequency threshold, and $S$ becomes

where

$$
S=S_{\mathbf{B}}+S_{\mathrm{S}}
$$

$$
S_{\mathrm{B}}=-\theta\left(\omega^{2}-l^{2} \kappa^{2}\right) \frac{\rho \kappa^{2}}{2 \pi \gamma} \frac{\left[\left(\omega^{2} / l^{2}\right)-2 \kappa^{2}\right]}{\left[\left(\omega^{2} / l^{2}\right)-2 \kappa^{2}\right]^{1 / 2}\left[\kappa^{6}-\left(\omega^{2} / l^{2}\right) \kappa^{4}-\rho^{2} \omega^{4} / \gamma^{2}\right]}
$$




$$
S_{\mathrm{S}}=\delta\left(\omega^{2}-\omega_{\mathrm{s}}^{2}\right), \quad \omega_{\mathrm{s}}=(\gamma / \rho) \beta_{l} \kappa^{1 / 2},
$$

$\theta(x)$ being the unit step function and $\omega_{\mathrm{s}}$ the frequency of the surface mode.

These are the three terms found in [1]. The contribution found in [2] comes from the bulk frequency threshold and does not take into account the surface mode contribution $\left(S_{\mathrm{S}}\right)$ and the distortion of the continuum of bulk states due to the existence of the surface $\left(S_{\mathrm{B}}\right)$.

On the other hand, from $\operatorname{Im} \mathcal{G}_{\mathrm{s}}$ one readily obtains the surface projected spectral function which is easily seen to be a particular case (inviscid fluid) of the formulae discussed in $[3,4,6]$.

Thus the results of [1], which determine the temperature dependence of the surface specific heat, are fully corroborated, while making contact with another important spectral function of physical interest.

\section{References}

[1] Djafari-Rouhani, B., Dobrzynski, L., J. Physique 43 (1982) 523.

[2] IosilevskiI, Ya. A., Phys. Rev. B 20 (1979) 1473.

[3] Loudon, R., Proc. R. Soc. London, A 372 (1980) 275.

[4] Loudon, R., in "Surface excitations ", Chap. 6, Modern problems in Condensed Matter Series, Vol. 9, V. M. Agranovich and R. Loudon, eds. (North-Holland, Amsterdam) 1984.

[5] Garcia-Moliner, F., Ann. Phys. 2 (1977) 179.

GARCIA-MOLINER, F., FloRes, F., "Introduction to the theory of solid surfaces » (Cambridge University Press) 1979.

[6] Platero, G., Velasco, V. R., Garcia-Moliner, F., Phys. Scr. 23 (1981) 1108.

[7] Velasco, V. R., Garcia-Moliner, F., Phys. Scr. 20 (1979) 111.

[8] Landau, L., Lifshitz, E. M., « Fluid mechanics » (Pergamon Press, Oxford) 1959. 